\title{
GAMMA DENSITOMETRY MEASUREMENTS OF GAS/ LIQUID FLOW WITH LOW LIQUID FRACTIONS IN HORIZONTAL AND INCLINED PIPES
}

\author{
S. VESTØL, W. A. S. KUMARA \& M. C. MELAAEN \\ University College of Southeast Norway, Porsgrunn, Norway.
}

\begin{abstract}
The ability to accurately predict the volume fraction of different phases flowing in a pipe is of extreme importance to nuclear and oil industries, among others. This article will focus on the application of a single-beam gamma densitometer to investigate gas/liquid flows with low liquid fractions in horizontal and inclined pipes. The experiments are performed in a $15 \mathrm{~m}$ long, $56 \mathrm{~mm}$ diameter, inclinable stainless steel pipe using Exxsol D60 oil (viscosity $1.30 \mathrm{mPa}$ s, density $793 \mathrm{~kg} / \mathrm{m}^{3}$ ), water (viscosity $0.89 \mathrm{mPa} \mathrm{s}$, density $999 \mathrm{~kg} / \mathrm{m}^{3}$ ) and air (viscosity $0.018 \mathrm{mPa} \mathrm{s}$, density $1.22 \mathrm{~kg} / \mathrm{m}^{3}$ ) as test fluids. The test pipe inclination is changed in the range from $5^{\circ}$ upward to $5^{\circ}$ downward. Experimental measurements are reported at three different mixture velocities, 5,10 and $15 \mathrm{~m} / \mathrm{s}$, and the inlet liquid fraction is varied from 0.0010 to 0.0100 . In the experiments, the vertical interface position is measured by traversing horizontal gamma beams, and the curvature of the interface is measured by traversing vertical and angular gamma beams.
\end{abstract}

Keywords: air/oil flow, air/water flow, gamma densitometer, mixture velocity, multiphase flow, volume fraction.

\section{INTRODUCTION}

Gamma-ray attenuation techniques have been widely used since the mid 1950s to measure phase volume fractions in simple adiabatic air-water flows and in high-pressure boiling flows [1]. Most of the reported phase volume fraction measurements are applicable for steady flows and excellent reviews on the subject are available in Schrock [2], Hewitt et al. [3], Hewitt [4] and Eberle et al. [5]. Gamma densitometer utilizes the concept of gamma attenuation in matter where the magnitude of attenuation is directly related to the density of the material through which the gamma ray passes as well as to the intensity of the ray itself. The advantage of gamma densitometry is that it is a non-intrusive technique, relatively inexpensive and is portable. Its main disadvantage is that the collimated beam of the gamma ray will produce a line averaged value and local information cannot be obtained. Another disadvantage has to do with the necessity to increase the strength of the gamma source with increase in the thickness and/or density of the pipe wall, which will require increased radiation protection and will reduce portability. The advantages and disadvantages of gamma densitometry applied to multiphase flow measurements are presented in detail by Babelli [1].

The available research on detailed phase fraction measurements in the gas/liquid twophase flows in pipes using gamma densitometry is limited in number and in nature. In the present work, single-beam gamma densitometry is used for non-intrusive measurements of phase distributions in gas/liquid flows in horizontal and slightly inclined pipes. In the experiments, the vertical interface position is measured by traversing horizontal gamma beams, and the curvature of the interface is measured by traversing vertical and angular gamma beams. 


\section{EXPERIMENTAL SET-UP}

\subsection{Multiphase flow loop}

A simplified flow sheet of the experimental rig is shown in Fig. 1, which has been presented in detail by Kumara et al. [6]. Three fluid phases are available in the multiphase flow facility: gas, oil and water. Oil and water are stored in separate tanks, T100 and T101, respectively. Seven different pumps (P100, P101, P102, P103, P104, P105 and P106) are available to circulate the liquid phases through the test system. Air is supplied from the central pressurized air system in the building, and the air flow rate is controlled with a pneumatic control valve (V131). The three fluid phases are mixed at the inlet of the test section that has been designed to initiate separated flow. The test section is made of steel, except for two short plastic sections. A $0.8 \mathrm{~m}$ long transparent acrylic section is used to allow visual observations of the flow while a $0.4 \mathrm{~m}$ long polypropylene section is used to allow measurements with the gamma densitometer. The length of the test pipe section is $15 \mathrm{~m}$ and the inner diameter is $56.3 \mathrm{~mm}$. A gas/liquid separator (R120) is located immediately downstream to the test section. The liquid outlet of the gas/liquid separator is connected to an oil/water separator (R100) located at a lower elevation where the oil and water phases are separated and returned to their respective storage tanks. Table 1 lists some important physical properties of the test fluids under atmospheric conditions. A controller based on LabView ${ }^{\circledR}$ is used for setting gas and liquid inflow rates and selecting appropriate pumps and flow meters. The flow rates are controlled by an automatic control loop, which uses the flow meter readings to adjust the pump speeds and valve openings.

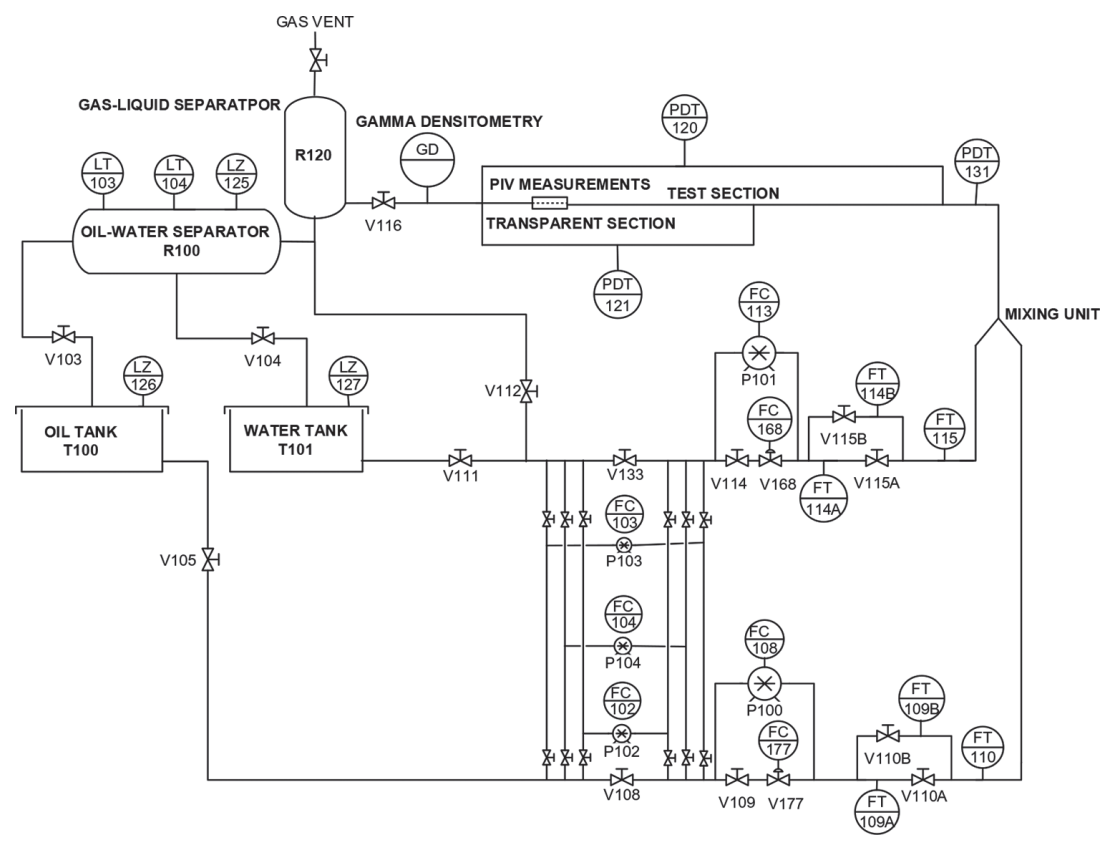

Figure 1: Simplified flow sheet of the test rig. 
Table 1: Physical properties of test fluids at atmospheric conditions.

\begin{tabular}{llll}
\hline & Gas & Oil & Water \\
\hline Fluid & Air & Exxsol D60 & Tap water \\
Density at $15^{\circ} \mathrm{C}$ & $1.22 \mathrm{~kg} / \mathrm{m} 3$ & $793 \mathrm{~kg} / \mathrm{m} 3$ & $999 \mathrm{~kg} / \mathrm{m} 3$ \\
Viscosity at $25^{\circ} \mathrm{C}$ & $0.018 \mathrm{mPa} \cdot \mathrm{s}$ & $1.3 \mathrm{mPa} \cdot \mathrm{s}$ & $0.89 \mathrm{mPa} \cdot \mathrm{s}$ \\
Surface tension at $25^{\circ} \mathrm{C}$ & & $25.9 \mathrm{mN} / \mathrm{m}$ & $72.0 \mathrm{mN} / \mathrm{m}$ \\
\hline
\end{tabular}

\subsection{Single-beam gamma densitometer}

The gamma densitometer consists of a radioactive source ( $45 \mathrm{mCi}, \mathrm{Am} 241)$, a detector (NaI) and signal analyzing system as shown in Fig. 2. The source and the detector are collimated so that only transmitted radiation will be detected and scattered radiation can be neglected. The source is located at one side of the pipe and sends a gamma beam toward the detector located at the opposite side of the pipe. The design, operation and measurement uncertainties of single-beam gamma densitometer measurements are presented in detail by Kumara et al. [6]. The gamma beams are traversed in order to measure the cross-sectional phase distribution. Vertical gamma beams and angular gamma beams are used to measure the curvature of the interface while horizontal gamma beams are used to measure the mean vertical interface position and to give some information about the interfacial waves. The beam paths used in the experiments are illustrated in Fig. 3.

The flows are allowed to stabilize for 5 min or longer depending on the flow characteristics before the measurements are started. The measurement time is $50 \mathrm{~s}$ for each measurement point. At least 95 measurement points are used for each flow condition and the total measurement time for one flow condition, including traversing time, is about $90 \mathrm{~min}$.

\subsection{Investigated flow conditions}

The mixture velocity, $\mathrm{U}_{\mathrm{m}}$, of gas/liquid flow is defined as follows.

$$
U_{m}=\frac{Q_{g}+Q_{l}}{A}
$$

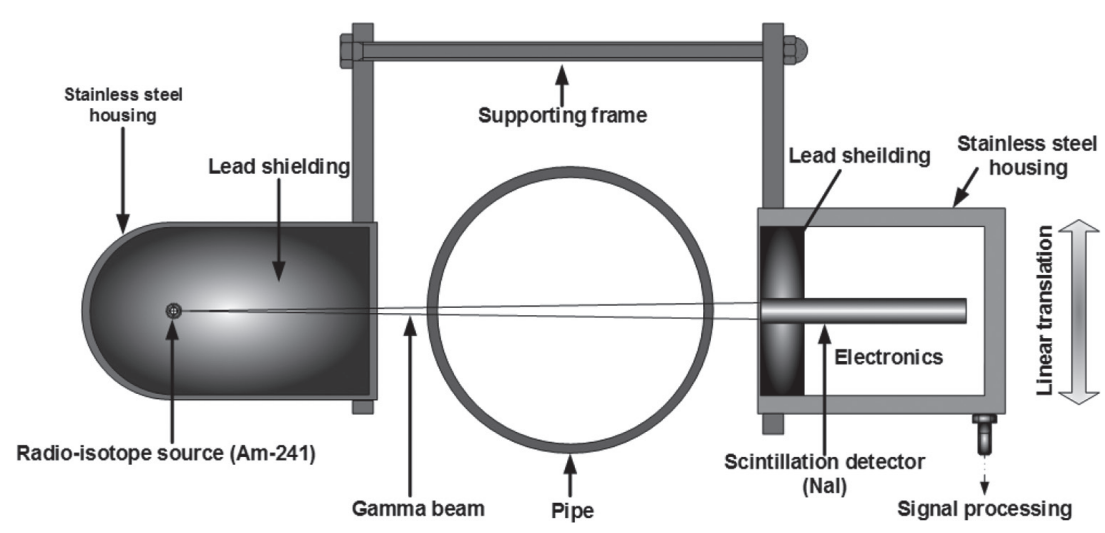

Figure 2: Single-beam gamma densitometer. 

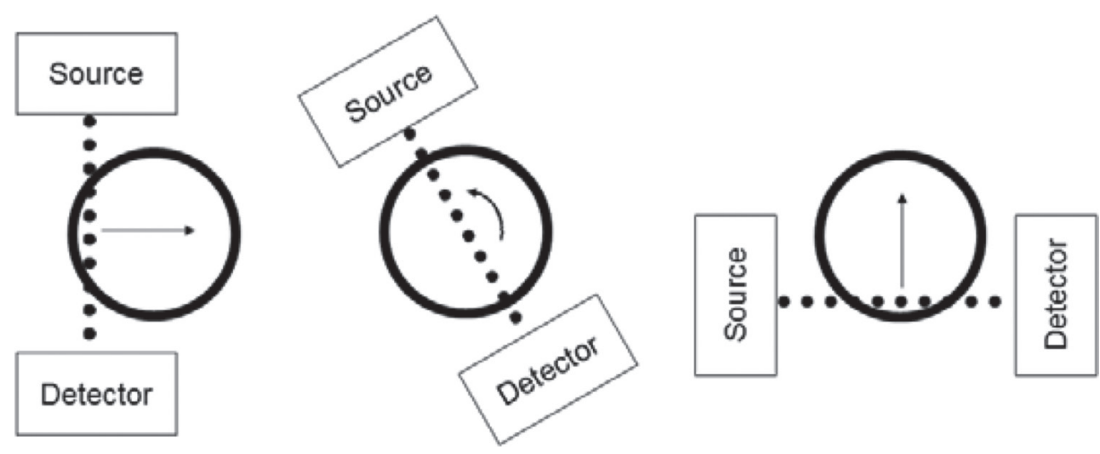

Figure 3: Illustration of gamma beam paths used in the experiments.

Where $Q_{g}$ and $Q_{l}$ are the inlet volumetric flow rates of gas and liquid, respectively, and $A$ is the pipe cross-sectional area. In the present work, mixture velocities of 5,10 and $15 \mathrm{~m} / \mathrm{s}$ are investigated.

The inlet liquid fraction, $\lambda_{l}$, is defined as;

$$
\lambda_{l}=\frac{Q_{l}}{Q_{g}+Q_{l}}
$$

The experiments are performed at five different inlet liquid fractions of $0.0010,0.0025$, $0.0050,0.0075$ and 0.0100 and at pipe inclinations of $-5^{\circ},-1^{\circ}, 0,+1^{\circ}$ and $+5^{\circ}$.

\section{RESULTS}

The phase distributions of gas/liquid flow in horizontal and near horizontal pipes are measured by traversing horizontal, vertical and angular gamma beams. The results are graphically presented below.

\subsection{Horizontal air/water flow with mixture velocity $5 \mathrm{~m} / \mathrm{s}$ and liquid fraction 0.0100}

Results from measurements with vertical and angular gamma beams on a horizontal air/water flow with mixture velocity $5 \mathrm{~m} / \mathrm{s}$ and liquid fraction 0.0100 are plotted in Fig. 4(a). In order to generate Fig. 4(a), it has been assumed that all the liquid flows at the bottom of the pipe and all the gas flows at the top of the pipe. This assumption is valid unless there is significant entrainment of gas in the liquid phase, liquid in the gas phase or liquid deposition on the pipe wall above the interface. Visual observations show that there is no entrainment or liquid deposition on the pipe wall in this flow and the flow pattern is observed to be stratified wavy flow without entrainment. The figure illustrates the average curvature of the interface. For this flow the interface is essentially flat except close to the wall where the interface curves a little upward. A good agreement is observed between the measurements with vertical and angular gamma beams. Measurements with vertical and angular gamma beams do not give any information about waves, entrainment or liquid deposition at the upper half of the pipe wall, but measurements with horizontal gamma beams can give some information about those parameters.

Figure 4(b) shows the liquid holdup, $h_{l}$, as a function of vertical pipe position for a horizontal air/water flow with mixture velocity $5 \mathrm{~m} / \mathrm{s}$ and liquid fraction 0.0100 . A still picture of 


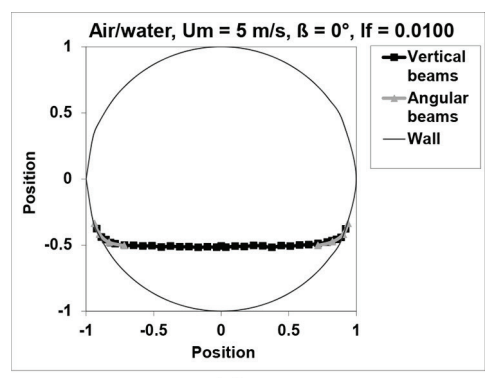

(a)

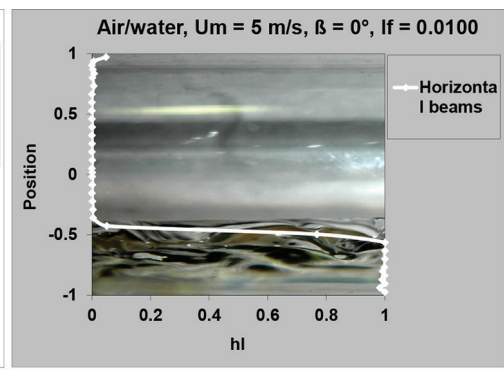

(b)

Figure 4: Measurements of holdup in horizontal air/water flow with mixture velocity $5 \mathrm{~m} / \mathrm{s}$ and liquid fraction 0.0100. (a) Vertical \& angular gamma beams; (b) Horizontal gamma beams.

the flow is put in the background of the figure. The plot gives the vertical interface position and some information about the interface structure. In this case, visual observations show that there is no entrainment of liquid in the gas phase, no entrainment of gas in the liquid phase and no liquid deposition on the pipe wall in this flow. Then the holdup measurement should be zero above the interface region and one below the interface region. In the interface region, the holdup measurement should be somewhere between zero and one because of the interfacial curvature and interfacial waves. The figure shows good agreement between the measurements and the image in the background of the figure except for the measurement point closest to the upper wall. In this point the measurements wrongly indicates a significant amount of liquid. This is explained by the reduced accuracy of the gamma measurements close to the pipe wall as described in Section 3.6. The observed error is caused by measurement noise and is well within the uncertainty limits. Please note that the interface position is highest near the wall, and this is the interface position that is clearly visible in the picture. This is higher than the interface position in the middle of the pipe; hence most of the interface line drawn in Fig. 4(b) is below the highest interface as seen in the picture.

Figure 5(a) is a plot of the measurements in Fig. 4(b) in a different coordinate system. Here, the measurements are plotted as the interface position in pipe coordinates and shows the liquid film thickness. It has been assumed that there is symmetry around the vertical axis

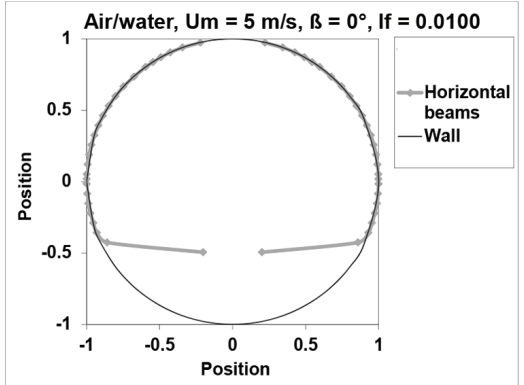

(a)

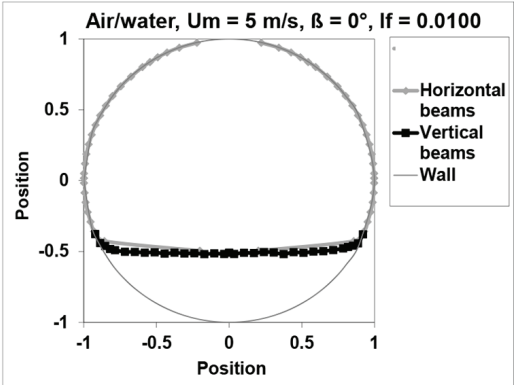

(b)

Figure 5: Measurements of holdup in horizontal air/water flow with mixture velocity $5 \mathrm{~m} / \mathrm{s}$ and liquid fraction 0.0100. (a) Interface position in pipe coordinates; (b) Vertical/ horizontal gamma beams 
through the pipe center and that all the liquid is in a film at the wall. This assumption is not valid in the wave area. Figure 5(a) shows that the error in the measurement closest to the upper wall is insignificant in terms of film thickness.

Measurements with horizontal gamma beams cannot discriminate between interfacial waves and interfacial curvature, but measurements with vertical gamma beams can be used to compensate for this. Measurements with horizontal gamma beams will be affected by the presence of waves in the interfacial area, but measurements with vertical gamma beams are practically unaffected by the presence of waves. Figure 5(b) shows a comparison of the measurements with vertical gamma beams from Fig. 4(a) and the measurements with horizontal gamma beams from Fig. 4(b). As can be seen from Fig. 5(b) the measurements with vertical gamma beams and the measurements with horizontal gamma beams agree quite well. This implies that the curvature of the interface is about the same size or larger compared to the interfacial waves. This agrees well with the visual observations that show that only small waves are present in this flow.

\subsection{Horizontal air/water flow with mixture velocity $15 \mathrm{~m} / \mathrm{s}$ and liquid fraction 0.0100}

Figure 6(a) shows results from measurements with vertical and angular gamma beams on a horizontal air/water flow with mixture velocity $15 \mathrm{~m} / \mathrm{s}$ and liquid fraction 0.0100 . This flow is observed to be in the transition flow pattern. It is mainly a stratified flow type where most of the liquid flows at the bottom of the pipe and the gas flows above it, but it also has elements from the annular flow and slug flow. In this flow pattern the wall is completely covered with a liquid film most of the time, like in an annular flow, but sometimes parts of the wall dries up and is only covered by droplets. The interfacial waves are large, and some of the waves reach all the way up to the top of the pipe and starts to form slugs.

It has been assumed that all the liquid flows at the bottom of the pipe according to Fig. 6(a) and similar to Fig. 4(a). This assumption is not fully satisfied since visual observations show that this flow has liquid deposition over the entire pipe wall as illustrated in Fig. 6(b). Since this cannot be detected with measurements with vertical or angular gamma beams, Fig. 6(a) is slightly misleading. It suggests that there is liquid only at the bottom half of the pipe, while in reality liquid covers the entire pipe wall. Visual observations and measurements with horizontal gamma beams are used to correct for this error. Since the volume of the liquid deposited

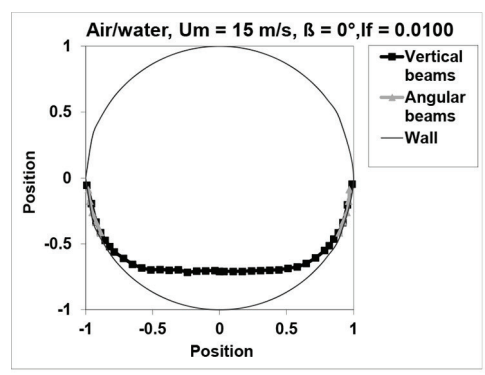

(a)

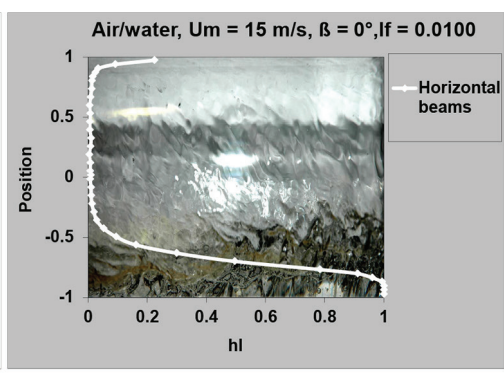

(b)

Figure 6: Measurements of holdup in horizontal air/water flow with mixture velocity $15 \mathrm{~m} / \mathrm{s}$ and liquid fraction 0.0100. (a) Vertical and angular gamma beams; (b) Horizontal gamma beams. 
at the upper half of the pipe wall is small compared to the volume of the liquid flowing at the bottom of the pipe, Fig. 6(a) gives a good description of the time averaged interface position in spite of this error. The figure shows that the interface curves more upward close to the wall compared to the corresponding flow case with mixture velocity $5 \mathrm{~m} / \mathrm{s}$; see Fig. 4(a). In addition, Fig. 6(a) shows good agreement between the measurements with vertical gamma beams and with angular gamma beams.

Results from measurements with horizontal gamma beams for a horizontal air/water flow with mixture velocity $15 \mathrm{~m} / \mathrm{s}$ and liquid fraction 0.0100 is presented in Fig. 6(b). The figure shows the time averaged holdup, $h_{l}$, as a function of vertical pipe position with a still picture of the flow in the background. Visual observations show that there is no entrainment of gas into the liquid phase in this flow, and the holdup below the interface region should be one. Above the interface region the holdup should be slightly above zero since there is some entrainment of liquid in the gas phase as well as some liquid deposition at the pipe wall above the interface. The measurements presented in Fig. 6(b) agree well with these expectations except for the measurement points closest to the upper wall where the holdup measurements are significantly larger than zero. This is partly caused by the decreased size of the measurement volume close to the upper wall while the liquid deposited at the pipe wall is more evenly distributed over the entire pipe wall. The liquid volume is then larger compared to the total measurement volume in the measurement points close to the upper wall than in the measurement points closer to the middle of the pipe, and the holdup is higher. The gamma beams cover a larger section of the wet wall in the measurement points closest to the upper wall; see Figs. 7(a) and 7(b). Another reason for the high holdup measurement close to the upper wall could be the increased measurement uncertainty close to the wall, see Section 3.6.

The holdup measurement in the measurement point closest to the upper wall is significantly larger than the measurement uncertainty in this measurement point, and gives a good indication of the presence of liquid at the pipe wall. The increased holdup measurement does not indicate a thicker liquid film at the top of the pipe, as can be seen when the results are plotted as interface position in pipe coordinates in Fig. 8(a). The interface region is much larger for this flow than for the corresponding flow with mixture velocity $5 \mathrm{~m} / \mathrm{s}$ presented in Fig. 4(b). This can be seen from the large region where the holdup is less than one and much larger than zero in Fig. 6(b). The large interface region could either be caused by waves or by interfacial curvature. Measurements with horizontal gamma beams are affected by both waves and interfacial curvature while measurements with vertical gamma beams are unable

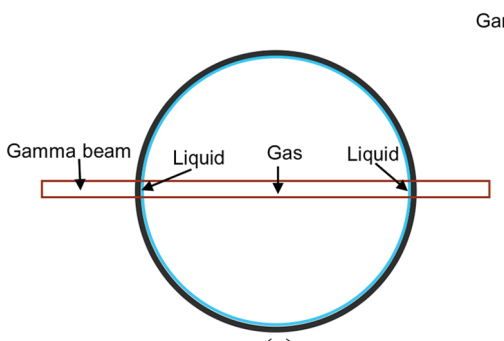

(a)

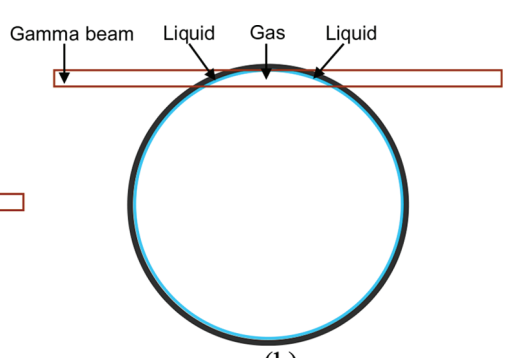

(b)

Figure 7: Illustration of liquid volume and gas volume for a measurement point. (a) At the middle of the pipe; (b) Close to the upper wall. 


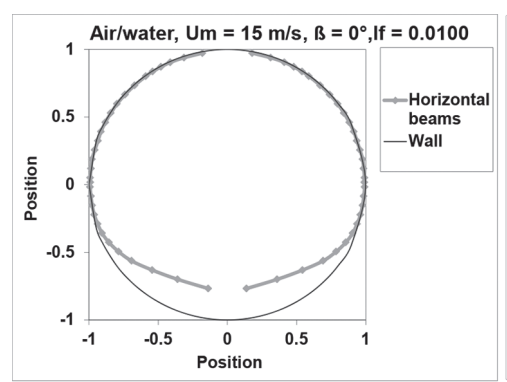

(a)

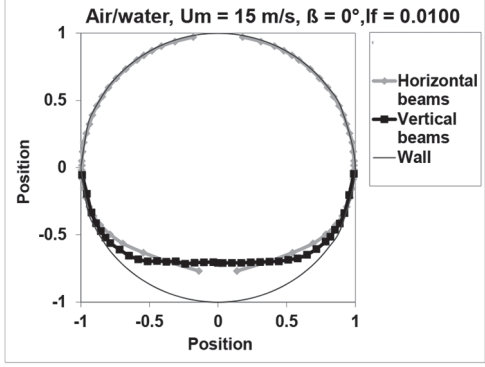

(b)

Figure 8: Measurements of holdup in horizontal air/water flow with mixture velocity $15 \mathrm{~m} / \mathrm{s}$ and liquid fraction 0.0100. (a) Interface position in pipe coordinates; (b) Vertical/ horizontal gamma beams.

to detect waves. In Fig. 8(b) the measurements with vertical and horizontal gamma beams are compared. As shown in the figure, reasonable agreement is observed between the measurements with horizontal and vertical gamma beams. In this case, large interfacial waves are observed compared to the interfacial curvature. The height of the interface area found from Fig. 6(b) (about 0.25 diameters) is approximately the same as the height of the largest waves.

\subsection{Horizontal air/water flows with mixture velocity $5 \mathrm{~m} / \mathrm{s}$ and different liquid fractions}

Gamma measurements of air/water flows with mixture velocity $5 \mathrm{~m} / \mathrm{s}$ and different liquid fractions are shown in Figs. 9(a) and 9(b). As expected, the holdup is observed to increase with increasing liquid fraction. The shape of the interface is similar for all the investigated liquid fractions. Figure 9(a) and 9(b) show that the interface is almost flat and only a small upward curvature is observed near the wall. In addition it shows that the interface region is thin and only small waves can be present. This is confirmed by visual observations where all these flow cases are identified as stratified wavy flow without entrainment. In this flow pattern only small smooth waves are present.

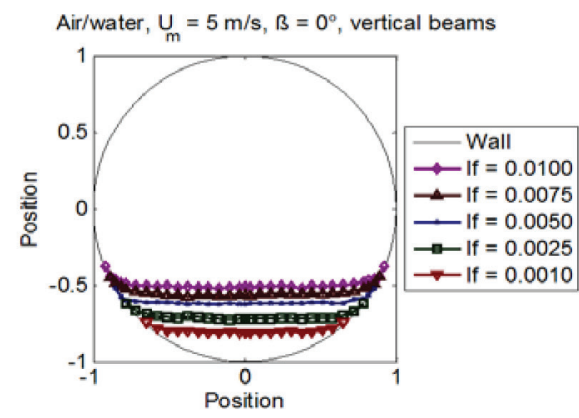

(a)

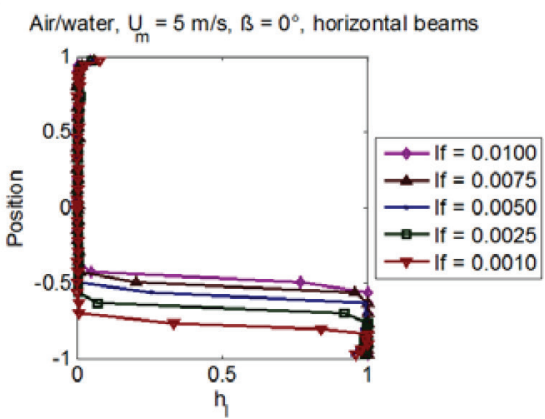

(b)

Figure 9: Measurements of holdup in horizontal air/water flow with mixture velocity $5 \mathrm{~m} / \mathrm{s}$ and different liquid fractions. (a) Vertical gamma beams; (b) Horizontal gamma beams. 


\subsection{Horizontal air/oil flows with mixture velocity $5 \mathrm{~m} / \mathrm{s}$ and different liquid fractions}

Figure 10 show gamma measurements for air/oil with the same conditions as for the air/water flows presented in Section 3.3. The air/oil flow results are very similar to the air/water results, but the air/oil measurements show slightly lower holdup for all these flow cases. The interface shape is practically the same for the air/oil flows as for the air/water flows. Visual observations show that the flow pattern changes within this measurement series of air/oil flows. The horizontal air/oil flows with a mixture velocity of $5 \mathrm{~m} / \mathrm{s}$ and liquid fraction 0.0010 and 0.0025 have been characterized as stratified smooth flow while the same flows with a mixture fraction of $0.0050,0.0075$ and 0.0100 have been characterized as stratified wavy without entrainment. This difference is not seen from the gamma measurements because these two flow patterns are too similar. The waves in the stratified wavy without entrainment flow pattern are too small to be seen by the gamma measurements.

3.5 Air/water flows with mixture velocity $5 \mathrm{~m} / \mathrm{s}$, liquid fraction 0.0100 and different pipe inclinations

Gamma measurements on air/water flows with mixture velocity $5 \mathrm{~m} / \mathrm{s}$, liquid fraction 0.0100 and different pipe inclinations are compared in Fig. 11. Under these conditions downward

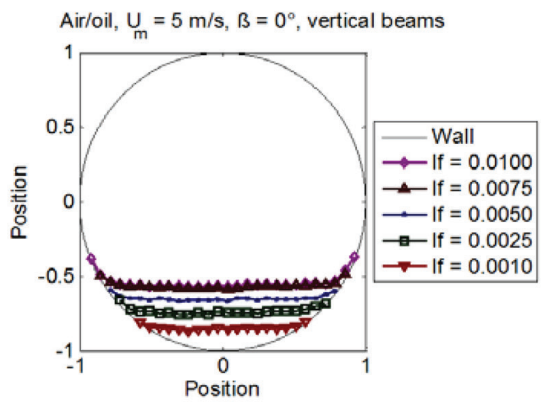

(a)

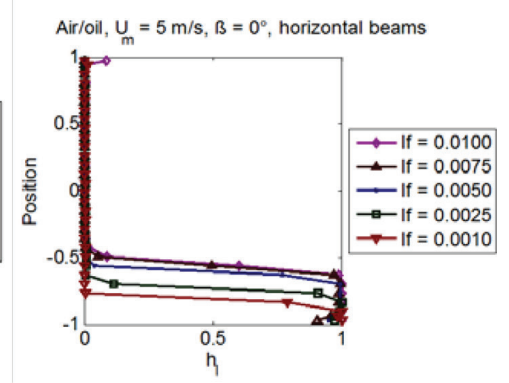

(b)

Figure 10: Measurements of holdup in horizontal air/oil flow with mixture velocity $5 \mathrm{~m} / \mathrm{s}$ and different liquid fractions. (a) Vertical gamma beams; (b) Horizontal gamma beams.

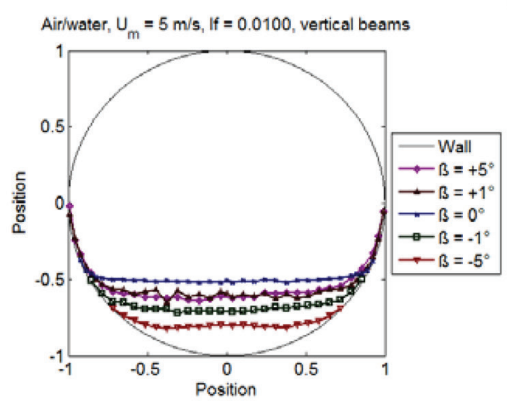

(a)

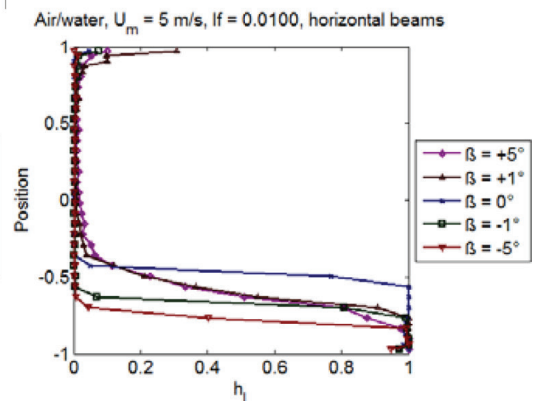

(b)

Figure 11: Measurements of holdup in air/water flows with mixture velocity $5 \mathrm{~m} / \mathrm{s}$ and different pipe inclinations. (a) Vertical gamma beams; (b) Horizontal gamma beams. 
inclinations and horizontal conditions give stratified wavy flow without entrainment while upward inclinations give slug flow. The gamma measurements cannot measure the transient effects which are dominant in slug flow as the results are time averaged values. The gamma measurement time for each measurement point is $50 \mathrm{~s}$. Based on visual observations, the slug frequencies are measured to about 8 and 19 slugs per minute for inclination $+1^{\circ}$ and $+5^{\circ}$, respectively. Thus, only a small number of slugs appear during the measurement time for each measurement point. Since the measurement time is not very long compared to the transient time scales in the system, a decreased accuracy is expected. This is the reason why the graphs for inclinations $+1^{\circ}$ and $+5^{\circ}$ in Fig. 11(a) are much more uneven than those for the rest of the measurements.

As shown in Fig. 11(a), measured time averaged liquid holdup is smallest for inclination $-5^{\circ}$, and it increases for inclination $-1^{\circ}$ and reaches a maximum at horizontal conditions. For upward inclinations, the time averaged holdup decreases again because of the change of flow pattern to slug flow. The differences in time averaged holdup between the flows with inclinations $+1^{\circ}$ and $+5^{\circ}$ are insignificant. The flow pattern change can be seen from Fig. 11(b). The interface region of the horizontal and downwardly inclined flows is small. This indicates that no large waves are present, which is typical for the stratified wavy flow without entrainments. For the upwardly inclined flows the interface region is large, indicating that large waves or slugs are present. An interesting observation that can be seen in Fig. 11(b) is that for the upwardly inclined flows the holdup in the upper part of the pipe is very low even though visual observations suggest that slugs cover the entire pipe cross-section. The reason for this is that the slugs move very quickly and are only inside the measurement volume for a small fraction of the measurement time, so the measurements of the liquid slugs have a small effect on the measured time averaged holdup. The fast movement of liquid in the slugs is also the reason why the time averaged holdup is lower for inclined flows compared to that for horizontal flows as can be seen from Fig. 11(a).

\subsection{Accuracy}

In gamma densitometry, the density is found from a frequency i.e. counts per second. From test measurements it is found that the measured frequency can vary by up to 200 counts per second for measurements of about 25,000 counts per second, i.e. $0.8 \%$ of the measured value. This is mainly caused by the inherent randomness in the emissions in the gamma source and the randomness in the absorption between the source and the detector. Two hundred counts per second correspond to an uncertainty in the holdup measurements of about 0.0075 for measurements in the middle of the pipe. For measurements close to the wall the uncertainty will increase because of the decreased control volume. In the measurement point closest to the wall the control volume is very small as a large part of the gamma beams hit the wall instead of the flow and the uncertainty in the holdup measurement can be approximately up to 0.15 . The random variations in frequency depend on the holdup and the relation between frequency and holdup is not linear.

The measurements with horizontal gamma beams can be used to find the interface height. Interface height is in the point where gamma measurements with horizontal gamma beams give a holdup of 0.5 . According to the analysis above, the relative accuracy of a holdup measurement of 0.5 is about $+/-1.5 \%$ if the interface is in the middle of the pipe and about $+/-30 \%$ if the interface is close to the bottom wall. The accuracy of the interface height measurement is limited more by the distance between each measurement point than the accuracy of each 
individual measurement point. The actual measurements will never be 0.5 . The true interface height must be found by interpolation between the two measurement points with a value closest to 0.5 . In the present experiments the distance between each measurement point is 2 $\mathrm{mm}$, so the accuracy of the interface height measurement will be the interpolated value +/$1 \mathrm{~mm}$. Based on this the average relative accuracy for all the interface height measurements with horizontal gamma beams is calculated to be $1.95 \%$. Measurements with horizontal gamma beams measure the average interface height. Since the interface is always curved upward by the walls, this will be slightly higher than the interface position in the center of the pipe. The measurements with horizontal gamma beams may also indicate if there is a liquid film or significant liquid entrainment in the gas part or gas bubbles in the liquid part of the pipe cross-section. The measurements with vertical gamma beams can be used to measure the interface position and the curvature of the interface. In general, higher measurement uncertainty is observed close to the pipe wall due to very low holdup and low control volumes where the interface meets the wall. For measurements in the middle of the pipe, the relative accuracy is better. The average relative uncertainty for the measurement points in the middle of the pipe is $+/-9.17 \%$. This is a much higher uncertainty than for the measurements with horizontal gamma beams of the average interface height. Since the interface is curved, the average interface height is higher than the interface position in the center plane of the pipe.

\section{CONCLUSIONS}

The cross-sectional phase distributions of air/water and air/oil flows in horizontal and near horizontal pipes are investigated using single beam gamma densitometry. The experiments are performed in a $15 \mathrm{~m}$ long, $56 \mathrm{~mm}$ diameter, inclinable stainless steel pipe. The test pipe inclination is changed in the range from $5^{\circ}$ upward to $5^{\circ}$ downward. Experimental measurements are reported at three different mixture velocities, 5,10 and $15 \mathrm{~m} / \mathrm{s}$, and the inlet liquid fractions is varied from 0.0010 to 0.0100 . In the experiments, the vertical interface position is measured by traversing horizontal gamma beams, and the curvature of the interface is measured by traversing vertical and angular gamma beams.

In horizontal flows at low mixture velocity $5 \mathrm{~m} / \mathrm{s}$, stratified flows are observed without entrainments and large waves. In stratified flows, the vertical interface position measured by horizontal gamma beams shows very good agreement with visual observations based on still flow images. In addition, interface curvature measurement based on angular and vertical shows a good comparison. However, reduced accuracy is observed close to the upper pipe wall.

Transition flow pattern is observed for horizontal gas/liquid flow at high mixture velocity, $15 \mathrm{~m} / \mathrm{s}$. It is mainly a stratified flow type where most of the liquid flows at the bottom of the pipe and the gas flows above it, but it also has elements from the annular flow and slug flow. Under these conditions, horizontal gamma beams measures the interface height accurately and shows good comparison with visual observations.

In general, measured liquid holdup for horizontal gas/liquid flow increases with inlet liquid fraction. Air/oil measurements show slightly lower holdup compared to the corresponding air/water flow cases. The interface shape is practically the same for both air/oil and air/water flows. The shape of the interface changes with changing liquid fraction at higher mixture velocities. Low liquid fractions give a small interface region and small waves, while higher liquid fractions give a larger interface region and large waves. In general, liquid holdup decreases with increasing mixture velocity.

The measured time averaged liquid holdup is smallest for inclination $-5^{\circ}$, and it increases for inclination $-1^{\circ}$ and reaches a maximum at horizontal conditions. For upward inclinations, 
the time averaged holdup decreases again because of the change of flow pattern to slug flow. For the air/water flows, the differences in the time averaged holdup profiles between the flows at $+1^{\circ}$ and $+5^{\circ}$ inclination is insignificant. It is important to note that the gamma measurements cannot measure the transient effects that are dominant in slug flow as the results are time averaged values.

The measurement uncertainties associated with single-beam gamma densitometer are also discussed. From test measurements it is found that the measured frequency can vary by up to 200 counts per second for measurements of about 25,000 counts per second, i.e. $0.8 \%$ of the measured value. This is mainly caused by the inherent randomness in the emissions in the gamma source and the randomness in the absorption between the source and the detector. For measurements close to the wall, the uncertainty will increase because of the decreased control volume.

\section{REFERENCES}

[1] Babelli, I.M.M., Development of the multiphase meter using gamma densitometer concept. Proceedings of International. Nuclear Conference, Kuala Lumpur, pp. 371-389, 1997.

[2] Schrock, V.E., Radiation attenuation techniques in two-phase flow measurements. 11th ASME/AICHE Heat transfer conference, Minneapolis, pp. 24-35, 1969.

[3] Hewitt, G.F. \& Lovegrove, P.C., Experimental methods in two-phase flow studies. Electrical power research institute report, EPRI-NP-118, 1976.

[4] Hewitt, G.F., Measurement of two-phase flow parameters, Academic Press, 1978.

[5] Eberle, C.S., Ishii, M. \& Revankar, S.T., A review of gamma densitometer designs and measurements in two-phase flows, Purdue University report, PU/NE-92-3, 1992.

[6] Kumara, W.A.S., Halvorsen, B.M. \& Melaaen, M.C., Single-beam gamma densitometry measurements of oil-water flow in horizontal and slightly inclined pipes. International Journal of Multiphase Flow, 36, pp. 467-480, 2010. https://doi.org/10.1016/j.ijmultiphaseflow.2010.02.003 